\title{
Enseñanza, lenguaje y pensamiento en cálculo. Un análisis cualitativo
}

\author{
Teaching, language and thinking in calculus. A \\ qualitative analysis
}

\section{Educação, linguagem e pensamento no cálculo. Uma análise qualitativa}

\author{
Sandra Liliana Zafra Tristancho' \\ Mawency Vergel Ortega² \\ José Joaquín Martínez Lozano3 \\ ${ }^{1}$ Policía Nacional de Colombia- Colombia
2 Universidad Francisco de Paula Santander - Colombia
${ }^{3}$ Escuela Superior de Administración Pública
Resumen
}

La investigación sigue un enfoque cualitativo método investigación acción. Su objetivo fue comprender qué sucede en el aula, cómo aprende el estudiante el cálculo apoyado de procesos de lenguaje. Involucra actores como estudiantes, docentes y padres de familia.

Fecha recibido: Agosto 17 de 2013

Fecha de aceptación: Diciembre 17 de 2013

DOI: http://dx.doi.org/10.22335/rlct.v5i2.386

1. Licenciada en Biología y Química, Especialista, maestría en prácticas pedagógicas. Filiación: Policía Nacional de Colombia. Correo: Sandra.zafra@correo.policia.gov.co

2. Licenciada en Matemáticas y Física. Especialista en Estadística Aplicada. Doctora en
Educación Filiación mawency@ufps.edu.co.

3. Licenciado en Biología y Química, Especialista en Biomatemática, Magister en Gerencia 3. Licenciado en Biología y Química, Especialista en Biomatemática, Magister en Gerencia
Educativa. Filiación: Escuela Superior de Administración Pública. Correo: correspondencia: Educativa. Filiacion:
checo.jf@gmail.com
Resultados: categorías explicativas fueron edad, tipo de institución, objeto matemático y complejidad semiótica asociada. Conclusiones: el alumno es el principal actor de su aprendizaje, la lectura como potenciadora del aprendizaje, proyectos para desarrollar competencias en la vida real, cooperar para aprender, juego para motivar, problemas para desarrollar habilidades y competencias.

Palabras clave: lenguaje, cálculo, docente, factores institucionales, valores

\section{Abstract}

Research follows a qualitative approach research action method. Its purpose was to understand what happens in the classroom, how the student learns the supported calculation of language processes. Involves actors such as students, teachers and parents. Results: explanatory categories were age, type of institution, mathematical object and associated semiotic complexity. Conclusions: the 
student is the main actor of his learning, reading as an enhancer of learning, projects to develop real life skills, cooperate to learn, play to motivate, problems to develop skills and competencies.

\section{Keywords:}

Language, calculus, teacher, institutional factors, values.

\section{Resumo}

Pesquisa segue abordagem à ação método de pesquisa qualitativa. Seu objetivo era entender o que acontece na sala de aula, como o aluno aprende o cálculo idioma suportado dos processos. Envolve atores: como alunos, professores e pais. Resultados: Foram explicativo idade categorias, tipo de instituição, objeto matemático e complexidade semiótica associada. Conclusões: O aluno é o principal jogador de sua aprendizagem, a leitura como um potenciador de aprendizagem, projetos de desenvolvimento de habilidades da vida real, cooperar para aprender, jogar para motivar, problemas para desenvolver habilidades e competências

\section{Palavras-chave:}

linguagem, cálculo, professor, fatores institucionais, valores.

\section{Introducción}

Instituciones en el ámbito mundial han trabajado en métodos, enfoques, técnicas y herramientas para mejorar el aprendizaje del lenguaje, del cálculo, de su comprensión y aplicación, sin tener en cuenta cómo funciona el cerebro. Se han esforzado algunas por transmitir informaciones para que sean recopiladas por los estudiantes, desconociendo que la mente es extremadamente deficiente para almacenar datos.

Investigadores han señalado que el cerebro está diseñado para crear, soñar, amar, inventar, procesar, analizar e interpretar la información, pero no para almacenarla. Para ello fueron creadas las nuevas tecnologías. Sin embargo, hasta ahora no se ha inventado algo que analice e interprete mejor la información que el cerebro humano, posiblemente nunca se podrá hacer con la flexibilidad, plasticidad y adaptabilidad que lo caracterizan"

En esta sociedad del conocimiento apoyado en tecnologías de la información y comunicación cabe entonces preguntarse qué hace exitoso a un estudiante de cálculo? o mejor, dado que no hemos encontrado una solución Universal. Donde está el problema en la Enseñanza del Cálculo. El problema está en el alumno?, el problema está en el maestro? O es en el Sistema Educativo donde se encuentran todas las problemáticas que no conllevan a un Aprendizaje Significativo.

Es relevante aceptar que cada día es mayoritario el uso de las tecnologías de la información y comunicación viviendo en una sociedad que posee una red casi ilimitada de circulación de archivos siendo este hecho permite la caracterización el desarrollo de la competencia para interpretar y analizar datos, como la meta cognitiva más importante del proceso educativo durante la media educacional.

En términos generales no se requiere tener en la cabeza la información exacta sobre los accidentes geográficos, los presidentes, los algoritmos, la gramática o los símbolos químicos, como había supuesto la escuela tradicional. Ahora bastará con una tecla de un computador o un celular para acceder a cualquier información necesaria. De la misma manera que hoy en día no tenemos que recordar los números telefónicos ya que éstos se pueden archivar en nuestros Smartphone. A propósito, ¿cuántos números telefónicos sabe usted si se le pierde el celular?. Encontramos ya estudiantes que resuelven ecuaciones, grafican, dan solución a Ecuaciones a través de los Apps en sus dispositivos móviles Lo anterior pone de manifiesto que necesitamos una gran variedad herramientas y aplicaciones que mejoren las posibilidades de comunicación donde al encontrar dicha información el joven la interprete, la analice y la constate con otras de diversas formas que trabaje hipotética y deductivamente con ella es decir desarrolle competencias en argumentar, deducir ,inferir e interpretar. Así como los deportistas necesitan ejercitar sus músculos para desarrollarlos, niños y jóvenes tienen que ejercitar una y otra vez sus procesos para pensar. La escuela debería ser un 
lugar para ejercitar estos procesos de pensamiento en todas las clases, en todos los cursos y en todas las asignaturas. La escuela tendría que ser un gimnasio para pensar.

Sin embargo, por dedicarse a transmitir múltiples informaciones desarticuladas, los niños y jóvenes en América Latina adquieren muy pocos conceptos de las ciencias humanas y básicas. Es por ello que cuando nuestros estudiantes son evaluados en lectura, en conceptos científicos y en resolución de problemas, América Latina se ubica en la cola del mundo y Colombia, tristemente, nos encontramos en los últimos lugares.

\section{Análisis}

Análisis de entrevistas realizadas a padres de familia con título de doctorado y maestría de niños y jóvenes de instituciones públicas y privadas de la ciudad de Cúcuta, muestran cómo las nuevas metodologías están cambiando los entornos educativos e impulsando mejores resultados académicos en sus hijos. Padre1 señala "Hoy día hay modelos innovadores", Padre2 "mi hijo dice que el profe le guía", Padre 4"el profesor le ayuda a descubrir".

Sucesos en el aula. Como podría analizarse entonces el proceso que vivencia realmente un joven en el aula, el cualquier grado básicamente sin hacer mayor esfuerzo observamos que el joven recibe los datos, los almacena en una primera fase que podríamos llamar preparación de datos, una segunda fase de Análisis inicial, donde encontramos una codificación y un análisis principal que contempla que el joven en el refinamiento del sistema escriba anotaciones, procesa, interpreta en el momento, en el sitio, hora y lugar en el cual pasan los acontecimientos o en el sitio o el lugar en el que el docente le presta las diferentes herramientas para que resuelva problemas. Entonces, el joven repasa, aprende, culmina su asignatura o modulo o contenido programático según sea en donde estudie y el nombre que le haya dado la institución a los contenidos, pero siempre de una manera asignaturista que lleva al joven después de un corto periodo de tiempo a olvidar, es decir, que lo lleva a un aprendizaje a corto tiempo .
Tenemos un estudiante que recopila la información, guarda como un medio magnético, solo memoriza, alcanza a analizar algunos preconceptos pero no adquiere hábitos de estudio, donde no se le tienen en cuenta sus intereses, siendo un joven centrado en el alcance de los videojuegos, en el aprendizaje de comunicación a través de la Red y muchas veces en el aprendizaje autónomo pero descontextualizado con la realidad que se vive en la Enseñanza del Cálculo.

Papel del profesor el aula

Se tiene un docente que transmite conocimiento pero que esta desactualizado, que es muy cómodo, poco innovador y se requiere adoptar una perspectiva pragmatista y que defina el significado de objeto matemático en términos de prácticas en donde resulte que el significado de un objeto matemático quede ligado a otro significado y a otros objetos puesto que las practicas interviene dicho objeto conjuntamente con otros objetos matemáticos, este hecho permitiría distinguir entre lo que interpretan los jóvenes como sentido y significado en un contexto de matemática educativa. En efecto puesto que el objeto se puede relacionar con uno $u$ otros objetos según el contexto, el tipo de notación da a lugar a diferentes prácticas entiende el sentido como un subconjunto del sistema de prácticas que constituye el significado del objeto, pero este significado de objeto matemático entendido como sistema de prácticas que se pueden parcelar en diferentes clases más específicas utilizadas en un contexto y con un determinado tipo de notación que le otorga un determinado sentido, teniendo en cuenta claro que cada contexto ayuda a producir el sentido, es decir permite generar un subconjunto de prácticas 


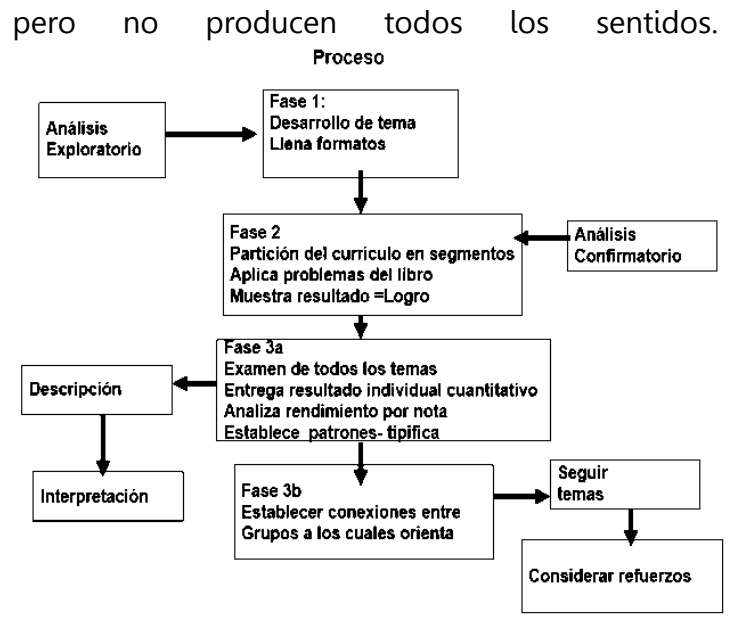

Figura 1. Fases desarrolladas en el aula

Es así como un objeto matemático originado como un emergente del sistema de prácticas permite resolver un determinado campo de problemas que con el paso del tiempo queda enmarcado en diferentes programas o proyectos de investigación. Cada nuevo programa de investigación permite resolver nuevos tipos de problemas, aplicar nuevas técnicas, relacionar el objeto y por tanto definir dicho de una manera diferente permite utilizar nuevas representaciones de esta manera con el paso del tiempo aparecen nuevos sistemas de prácticas, nuevos sentidos que amplían el significado del objeto. El significado de un objeto personal se entiende como el sistema de prácticas matemáticas personales que una persona realiza para resolver el campo de problemas del cual ha emergido el objeto. Esta manera de entender los objetos y sus significados supone que la institución o la persona disponen de prácticas con respecto al campo de experiencia que el objeto abarca.

Las prácticas en las que prima el componente operatorio o actuativo nos permite realizar acciones y argumentaciones cuya finalidad es la resolución de situaciones problema, las practicas discursivas comunicativas están relacionadas con el dominio y la creación del lenguaje así como su uso para la realización de argumentaciones que permitan dar una justificación de la validez de las acciones realizadas, las practicas regulativas, normativas están orientadas básicamente a conseguir establecer propiedades, proposiciones y definiciones de conceptos; la reflexión anterior sobre las practicas hace necesario ampliar lo que se entiende por objeto matemático y nos limitamos a los conceptos EOS, Objetos matemáticos se obtiene el objeto en algunos de los siguientes elementos: lengua, acción, argumentación, concepto, propiedades, situación problema, cada uno de estos elementos excepto las situaciones problema, se puede entender como un emergente de las prácticas cuya finalidad es la relación de situaciones problema, a su vez la situaciones problema se pueden entender como emergentes de otros tipos de prácticas, necesidad de contextualizar y aplicar las matemáticas ,necesidad de generalizar, necesidad de resolver problemas.

En lo dicho anteriormente se ha considerado los objetos que emergen de las prácticas, ahora bien a su vez la realización de cualquier práctica es necesaria activar un conglomerado formado por elementos como la institución, sus normas, prácticas, enfoque, herramientas didácticas, clima organizacional y variables asociadas al aprendizaje (Figura 1), el lenguaje, las situaciones, los conceptos, propiedades, acciones y argumentaciones.

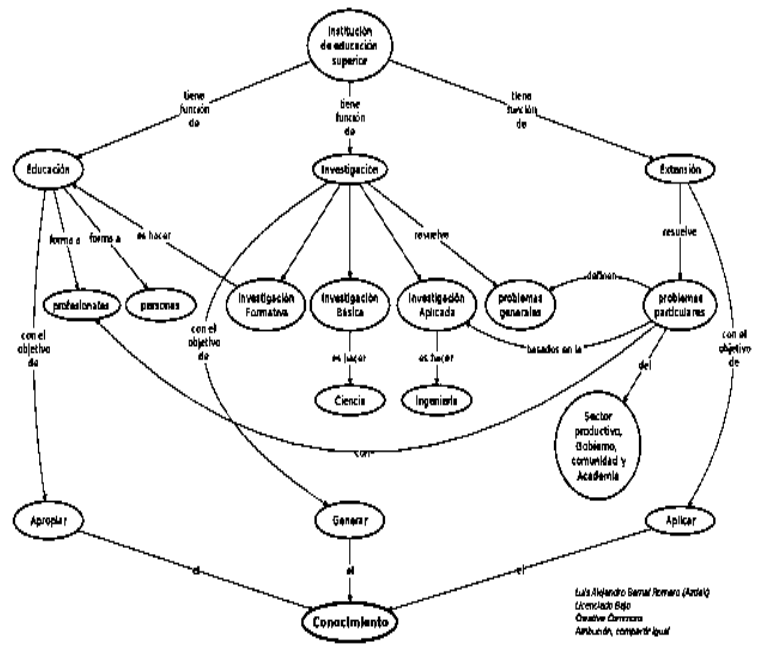

Figura 2. Variables asociadas a la enseñanza del cálculo. 
Para realizar una práctica matemática por ejemplo la representación gráfica de una función el sujeto necesita una serie de conocimientos sobre la representación gráfica de funciones que son fundamentadas para:

1) La realización de la práctica que consiste en representar una función determinada.

2) Para la interpretación de sus resultados como satisfactorios, es decir que hay que tener en cuenta que el sujeto tiene un conocimiento sobre la representación gráfica; por ejemplo como resultado del proceso de instrucción. También podemos considerar que tiene unas capacidades y habilidades de tipo general.

Así mismo existen otras problemáticas presentes en el desarrollo del pensamiento en cálculo y de la comprensión de textos y problemas, en el cual el joven como persona interviene, algunos estudios centrados en investigar la presencia de problemas comportamentales y emocionales ha seguido varias estrategias que se centran en comportamientos pero están aislados de las Instituciones Educativas y de la realidad que vive el joven en esta institución en su proceso de aprendizaje. Este dato ha recibido diferentes interpretaciones planteándose incluso hipótesis de mayor tendencia o sensibilidad que incorporan a la familia en el proceso educativo, a fin de buscar recursos o analizar esto de manera externa ante las dificultades que se presentan en el proceso de enseñanza-aprendizaje del Cálculo en el joven.

Estudios más recientes indican que la mayoría de jóvenes están psicológicamente sanos pero que presentan dificultades de atención, concentración y no presentan hiperactividad sino la ausencia de atención de los padres o los maestros los sienten ausentes dentro del proceso educativo. Una proporción importante manifiesta agresividad en su relación con otros o aislamiento social y esto se une a otras problemáticas como el uso de sustancias psicoactivas, así mismo se encuentran problemáticas asociadas al rendimiento académico o logros en el desarrollo del cálculo dado el desconocimiento de las capacidades, competencias, habilidades y en la etapa del
Desarrollo del Pensamiento en la cual se encuentra el joven.

El análisis Ontosemiótico al resaltar el papel que podría tener determinados usos de temáticas de cálculo en diferentes fenómenos usa de manifiesto la magnitud que podría llegar a tener este fenómeno en los países en los que este tipo de notación tuviese un papel predominante, de esta manera dirigir investigaciones posteriores como la de Badillo (2003), así lo mostró una investigación sobre la función derivada realizada con profesores colombianos en la cual la característica explicada y que por lo tanto ha de ser problematizada es la dificultad observada en la comprensión que tienen los alumnos de este objeto: "función derivada" manifestada en conflictos semióticos.

Las variables explicativas son: edad, tipo de institución, objeto matemático y complejidad semiótica asociada (Figura 2). Documentando que dicho fenómeno en Colombia no se limita a los alumnos sino que es de mayor magnitud ya que en muchos casos son los profesores los que confunden la derivada en un punto y la función derivada; dicho de otra manera la técnica que la institución escolar pretende que apliquen los alumnos el conocimiento, solo es posible si se traduce una configuración epistémica en la que el componente lenguaje contempla la representación gráfica y la simbólica conjuntamente, sino se contempla la representación gráfica la técnica no es viable. Por tanto contemplar la representación gráfica además de la simbólica permite realizar determinadas prácticas que con solo la representación simbólica no sería posible. Ahora bien es importante resaltar que no conviene focalizar la tensión solo en la relación de dos componentes de la configuración epistémica: lenguaje y técnica. Y olvidar los demás componentes puesto que estos son también son esenciales es necesario que la derivada se halla definida como pendiente de la recta tangente de acuerdo a su definición. Que se acepte una documentación basada en la observación visual de las que subtangentes midan todo lo anteriormente dicho de la deriva Proceso del maestro en el aula.

Si nos detenemos a pensar cual es el proceso que vivencia un maestro en el aula actualmente, será que los estudiantes alcanzan a interpretar a crear 
constructos?, pues bien sin detenernos en objeto, constructo, sentido y significado, Vergel, Rincón y Martínez (2012), en una investigación sobre prácticas pedagógicas analizando las fases donde observaron en una primera fase que incluía un análisis exploratorio, en este análisis los docentes desarrollaban los temas llenando una cantidad de formatos exigidos por la institución educativa y luego pasaban a una segunda fase que comprendía la partición del Currículo en segmentos para poder cumplir con todas las temáticas planteadas en lo que hoy llaman las instituciones de Educación
Figura 3. Factores asociados al lenguaje y comprensión de problemas en cálculo

A profesores cuyos padres cualificaron como positivas sus prácticas al respecto se preguntó a profesores de niños cuyos padres mencionaron expresiones asociadas a tecnologías y modelos innovadores, quienes dieron respuestas cómo:

Prof1"Yo me baso en el aprendizaje basado en proyectos" Prof5"Porque el alumno es el actor del

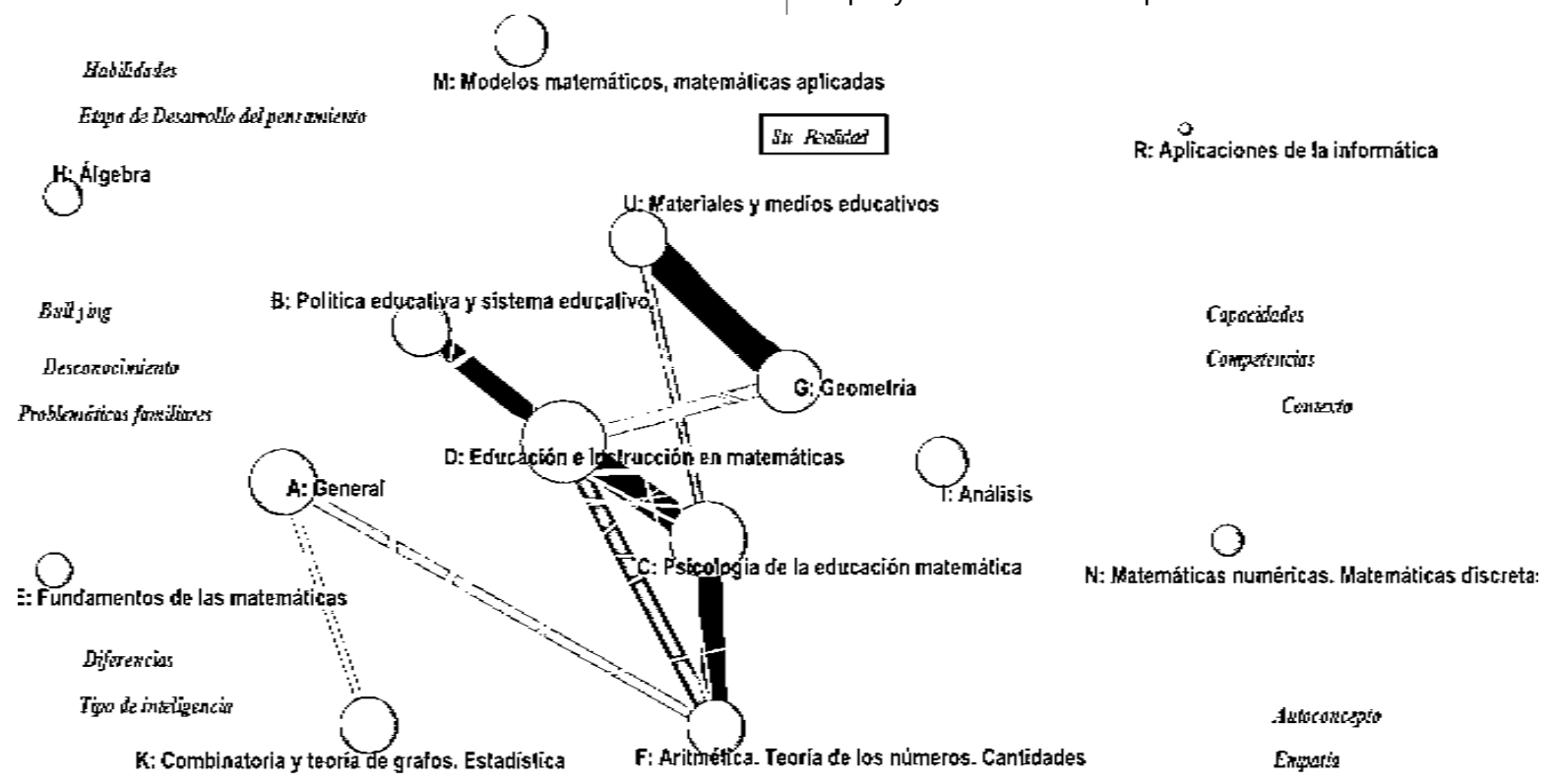

p. Informatirina

Superior en Colombia Syllabus, y a partir de allí lograban algunos aplicar problemas de textos y mostrarlo finalmente los resultados lo que se llamaban logros en los estudiantes realizando con esto un análisis confirmatorio de lo vivenciado en sus clases. Una tercera fase comprendía el examen de cada uno de los temas o finalmente el examen de todos los temas donde entregaban un resultado individual cuantitativo a los jóvenes, analizaban el rendimiento por notas y establecían patrones tipificando a cada uno de sus estudiantes respecto a su rendimiento académico para así generar estadísticas que conllevaran a la deserción o determinar la permanencia de cada uno de los jóvenes en cada una de sus clases. proceso" Prof 7 "amo mi trabajo", Prof9 "el estudiante es el alma del aula".

Prof 2 "el juego, me baso en el juego", "les gusta"

Prof8 "doy a mis alumnos temas antes de ser vistos, por guías, lecturas o blogs", "luego los trabajo en clase"

Prof 3. "me baso en problemas" "desarrolla sus

habilidades"

Prof4. "identifico problemas individuales" "los agrupo" "trabajan en problemas", "diseñan". 
Prof 5: "busco desarrollar su competencias"

Prof 9 "implemento aprendizaje cooperativo", los desarrolla competencias sociales, "se organizan en grupo", "se relacionan", "comparten" "se comprometen"

Prof 10" me baso en proyectos" "ven lo útil del cálculo" "interpretan".

El alumno actor de su aprendizaje, la lectura como potenciadora del aprendizaje, proyectos para desarrollar competencias en la vida real, cooperar para aprender, juego para motivar, problemas para desarrollar habilidades y competencias.

Esa fase también comprendía establecer conexiones entre grupos a los cuales orientaba, en esta fase el maestro no solo analizaba lo que pasaba en un aula de clase sino lo que pasaba en sus diferentes clases; sin embargo el maestro seguía orientando temáticas considerando refuerzos donde el estudiante no lograba alcanzar los aprendizajes "significativos" y realizando con esto básicamente una descripción o caracterización de los jóvenes, pero no llegaba a la interpretación de las diferentes temáticas que estaba vivenciando en su clase; es decir no estamos generando conocimiento $\mathrm{o}$ el estudiante no estaba entendiendo su objeto matemático; su significado, no le estaba dando un sentido a este significado en el contexto que la matemática requiere.

Proceso general de enseñanza El sistema educativo

El sistema educativo está pensado para transmitir informaciones y no para pensar. Así fueron pensados los currículos, los sistemas de evaluación, la selección y formación de los maestros. Así también están pensados los museos, hasta los concursos y noticieros de televisión. Han sido construidos para transmitir informaciones, pero no para interpretarlas, analizarlas o leerlas de manera crítica e independiente. Exige al maestro cumplir con programas estáticos

-Entregar documentos

-Protocolos de asistencia
-No cuenta con infraestructura tecnológica necesaria para estar acorde a la realidad que viven los jóvenes en el siglo XXI

\section{Referencias bibliográficas}

Almario García, Ó. (2007), Los sujetos colectivos en la formación del Estado nacional colombiano Medellín: Universidad Nacional de Colombia, 64p.

Alpizar E.; E. Dechamp, F. Lapeyren Montes, C. Guilhaumon, B. Baertrand, C. Jourdan. et al. (2008). Agrobacterium rhizogenes-transformed roots ofCoffee (C. arabica): Conditions for longtermproliferation and morphological and molecularcharacterization. Ann Bot 101 (7). 929940.

Anderson, E. (2011). Democracy, Public policy, and lay assessments of scientific testimony. Episteme 8 , 2, Cambridge, Universidad de Cambridge, 144.

Azen, S. P. y A. A. Afifi. (1972). Asymptotic and smallsample behavior of estimated Bayes rules for classifying time-dependent observations. Biometrics, 28, 989-998.

Careri, F. (2002). El andar como práctica estética. Barcelona: Editorial Gustavo Gili, 45.

Cardona Zuluaga, Alba Patricia (2014). "Memoria, palabra y acción: La historia patria, un saber para el sentimiento". En Anuario de Historia Regional y de las Fronteras. 21, 2, Bucaramanga, Universidad Industrial de Santander, 19.

Contreras Bello, Y. (2011). ¡Hasta la vista, baby!: Un ensayo sobre los tecnopensamientos. Revista Logos Ciencia \& TecnologíA, 3(1), 236-239. Recuperado de http://revistalogos.policia.edu.co/index.php/rlct/art icle/view/143/155

Contreras Bello, Y. (2012). Los elementos de la investigación: como reconocerlos, diseñarlos y construirlos. Autor: Hugo Cerda Gutiérrez. Colombia: Editorial Magisterio, 2011, 521 pp. Revista Logos Ciencia \& TecnologíA, 4(1), 220-221. Recuperado de http://revistalogos.policia.edu.co/index.php/rlct/art icle/view/183/194 
Contreras Bello, Y. (2013). El largo camino hacia la paz. Autores: Farid Samir Benavides

Codina Canet, M. A., Olmeda Gómez, C., \& Perianes Rodríguez, A. (2013). Analysis of Scientific Production and Research Specialization of the Universidad Politécnica de Valencia, Scopus (20032008), 36(3), $\quad 17 \quad p$ https://doi.org/10.3989/redc.2013.3.942

Fontana, J. (2001), La historia de los hombres. Barcelona: Crítica, 23p.

Herrera, M.C. (2007), "El memorial de las identidades: entre héroes y villanos. En la busca de sí y de los demás también", en Revista Folios, 25, Bogotá, Universidad Pedagógica Nacional, 53p.

Guerreiro O.; P. Denolf, M. Peferoen, B. Decazy, A. Eskes and R. Frutos. (1998). Susceptibility of the Coffee Leaf Miner (Perileucoptera spp) to B.thuringiensis $\square$-endotoxins: A model for transgenic perennial crops resistant to endocarpic insects. Current Microbiology 36. 175-179

Guitian, Dyna, "Los Bienes Culturales en el Espacio Habitable", en Revista Argos, 24, 47, Caracas, Universidad Central de Venezuela, 2007, 28.

Koselleck, R. (2012) Historias de conceptos. Estudios sobre semántica y pragmática del lenguaje político y social Madrid: Trotta, 89p.

Loaiza Cano, G (2011). Sociabilidad, religión y política en al definición de la nación: Colombia 1820-1886. Bogotá: Universidad externado de Colombia, 160.

López Lopera, L. (2014) "Figuraciones de la tierra natal: patria nación y república", en Coherencia, 11, 21, Medellín, Universidad EAFIT. 97p.

Medina, A. (¡994). Cita Histórica Tomo II. San José de Cúcuta: Cámara de Comercio de Cúcuta. 30p.

Martínez Osorio, P. (2012). La enseñanza en arquitectura y las visiones normativas hegemónicas, desde el contexto global al local. Revista Logos Ciencia \& TecnologíA, 4(1), 121-129. Recuperado dehttp://revistalogos.policia.edu.co/index.php/rlct/ article/view/174/185
Quijada, M. (1994). “¿Qué Nación? Dinámicas y dicotomías de la nación en el imaginario hispanoamericano del siglo XIX", en Cuadernos de Historia Latinoamericana, 2, Hamburg, Asociación de Historiadores Latinoamericanistas Europeos, $60 p$.

Graybill, F. A. (1976) Theory and Application of the Linear Model. Wadsworth Publishing Company. 70

Contreras Bello, Y. (2012). Los elementos de la investigación: como reconocerlos, diseñarlos y construirlos. Autor: Hugo Cerda Gutiérrez. Colombia: Editorial Magisterio, 2011, 521 pp. Revista Logos Ciencia \& TecnologíA, 4(1), 220-221. Recuperado de http://revistalogos.policia.edu.co/index.php/rlct/art icle/view/183/194

Contreras Bello, Y. (2012). Bases de la Investigación Cualitativa, técnicas y procedimientos para desarrollar una teoría fundamentada. Autores: Anselm Strauss y Juliet Corbin. Universidad de Antioquia, Colombia, 2012. Revista Logos Ciencia \& Tecnología, 3(2), 172-173. doi:http://dx.doi.org/10.22335/rlct.v3i2.166

Giménez, A.M.; Ríos N.A.(1999). Crecimiento de Schinopsis Quebracho colorado (Schlecht.) Barkl et meyer, Anacardiaceae. Madera y Bosques 5(2). 3551.

Kaweesa, S. H., Jonkvorst, R. J., Katebaka, R., Ssemmanda, R., Pomeroy, D., \& Brouwer, J. (2013). Is the Hamerkop Scopus umbretta a neo-colonist or an opportunist nester? Scopus, 32, 35-38.

Kanno T.; S. Naito and K. Shimamoto. (2000). Posttranscriptional Gene Silencing in Cultured Rice Cells. Plant Cell Physiol 41 (3): 321-326.

Leroy T.; A. Henry, M. Royer, I. Altosaar, R. Frutos and R. Phillipe. (2000). Genetically modified coffee plants expressing the $B$. thuringiensis cry $1 A C$ genefor resistance to leaf miner. Plant Cell Rep 19: 382-389.

Libiakova G.; B. Jorgensen, G. Palmgren, P. Ulvskor and E. Johansen. (2001). Efficacy of an introncontainingkanamicin resistance gene as a 
selectable marker in plant transformation. Plant Cell Rep 20: 610-615.

Littell, R. C.; G. A. Milliken; W.W. Stroup y R. D. Wolfinger. (1996).

SAS System for Mixed Models. Cary, N.C.: SAS Institute Inc.633pgs.

Miranda, H. I. C., Aguiar, P. R., Euzebio, C. D. G., \& Bianchi, E. C. (2010). Fuzzy logic to predict thermal damages of ground parts. Scopus, 434-441.

Núñez-Lagos Roglá, R. (2011). La radioactividad ambiental. Revista Logos Ciencia \& Tecnología, 2(2), 50-61.

doi:http://dx.doi.org/10.22335/rlct.v2i2.82

Pereira dos Anjos, M. (2010). El urbanismo en las ciudades latinoamericanas y la cooperación en la gestión urbana entre Brasil y Colombia. Revista Logos Ciencia \& Tecnología, 1(2), 152-156. doi:http://dx.doi.org/10.22335/rlct.v1i2.55

Ramos Moreno, A., Mayor Polanía, R., Ortiz P, N., \& Tovar Pérez, L. (2012). La diversidad en aves como factor determinante de la interacción entre ecosistemas del departamento del Huila. Revista Logos Ciencia \& Tecnología, 3(2), 45-58. doi:http://dx.doi.org/10.22335/rlct.v3i2.158

Ruiz-Sánchez, M.C., Torrecillas, A., DelAmor, F., León, A. y Abrisqueta, J.M. 1988. Leaf water potential and leaf conductance during the growingseason in almond trees under different irrigation regimes. Biologia Plantarum (30):327-332

Simonneau, T., Habib, R., Goutouly, J.P. y Huguet, J.G. 1993. Diurnal changes in stem diameter depend upon variations in water content: direct evidence in peach trees. Journal of Experimental Botany (44):615-621.

Syvertsen, J.P., Lloyd, J. y Kriedemann, P.E. 1988. Salinity and drought stress effects on foliar ion concentration, water relations, and photosynthetic characteristics of orchard citrus. Australian Journal of Agricultural Research (39):619-627.

Smallie, J., \& Virani, M. Z. (2010). A preliminary assessment of the potential risks from electrical infrastructure to large birds in Kenya. Scopus, 30, 32-39. https://doi.org/10.2193/2008-525

Rapoport, A (1978). Aspectos humanos de la forma urbana. Hacia una confrontación de las ciencias sociales en el diseño de la forma urbana. Barcelona: Gustavo Gili, 343p.

Rapoport, A (2003). Cultura Arquitectura y Diseño (Barcelona: Editorial Gustavo Gilli, 156p.

Smithson, R. (1991) Art Through the Camera's eye. New York: University Press, 130.

Tabuenca, M.C. y Herrero, J. 1966. Influencia de la temperatura en la época de floración de los frutales. An Aula Dei (8):115-153.

Tardieu, F. y Simonneau, T. 1998. Variability among species of stomatal control under fluctuating soil water status and evaporative demand: modelling isohydric and anisohydric behaviours. Journal of Experimental Botany (49):419-432.

Torrecillas, A., Domingo, R., Galego, R. y RuizSánchez, M.C. 2000. Apricot tree response to withholding irrigation at different phonological periods. Scientia Horticulturae (85):201-215.

Tunjano Huerta, C., \& Calvo Valencia, D. (2011). Evaluación de sustancias fitoprotectoras usadas como estrategia de neutralización de la acción del glifosato sobre cultivos de erythroxylum coca. Revista Logos Ciencia \& Tecnología, 2(2), 26-31. doi:http://dx.doi.org/10.22335/rlct.v2i2.79

Vanegas, A. y Ospina A.M. Colombia: Editorial Ibáñez, 2013, 423 pp.. Revista Logos Ciencia \& Tecnología, 4(2), 168-169. Recuperado de http://revistalogos.policia.edu.co/index.php/rlct/art icle/view/200

Vega, Felix. Conozcamos a Cúcuta (San José de Cúcuta: Cámara de Comercio de Cúcuta, 1993), 128.

Vanegas, A. y Ospina A.M. Colombia: Editorial Ibáñez (2013), Revista Logos Ciencia \& Tecnología, 4(2), 168-169. Recuperado de http://revistalogos.policia.edu.co/index.php/rlct/art icle/view/200 
Vélez, J.E., Intrigliolo, D.S. y Castel, J.R. 2007. Scheduling deficit irrigation of citrus trees with maximum daily trunk shrinkage. Agricultural Water Management (90):197-204.

Vonesh, E. y Chinchilli, V. (1997). Linear and nonlinear models for the analysis of repeated measurements. Statistic textbooks and Monographs, Inc. New York Ed. Marcel Dekker, 560

Witkin, S. (2011) Social construction and social work practice: interpretations and innovations. (New York: Columbia University Press, 2011), 87.

Wolfinger, R. D. y O'Connell, M. (1993). Generalized linear mixed models: a pseudo-likelihood approach. J. Statist. Comput. Simul. 48:233-243.

Yohannes, E., Nikolaus, G., \& Pearson, D. J. (2013). Stable isotopes of soil collected from feet of two species of migratory Acrocephalus give clues to stopover sites. Scopus, 32, 1-9. 